\title{
Culture and Leukocyte Adhesion Assay of Human Arterial Endothelial Cells in a Glass Microchip
}

\author{
Yo Tanaka, ${ }^{* 1, * 2}$ Yuji KikuKawa, ${ }^{* 1}$ Kae Sato, ${ }^{* 3}$ Yasuhiko SugII, ${ }^{* 4}$ and Takehiko KitamorI ${ }^{* 1, * 2, * 3, * 5 \dagger}$ \\ *1 Department of Applied Chemistry, Graduate School of Engineering, The University of Tokyo, \\ 7-3-1 Hongo, Bunkyo, Tokyo 113-8656, Japan \\ *2 Core Research for Evolutional Science and Technology (CREST), Japan Science and Technology Agency, \\ Kawaguchi, Saitama 332-0012, Japan \\ *3 Center for NanoBio Integration, The University of Tokyo, 7-3-1 Hongo, Bunkyo, Tokyo 113-8656, Japan \\ *4 Research Institute for Science and Technology, Kogakuin University, \\ 2665-1 Nakanocho, Hachioji, Tokyo 192-0015, Japan \\ *5 Micro Chemistry Group, Optical Science Laboratory, Kanagawa Academy of Science and Technology (KAST), \\ 3-2-1 Sakado, Takatsu, Kawasaki, Kanagawa 213-0012, Japan
}

\begin{abstract}
Cells are frequently exploited as processing components for integrated chemical systems, such as biochemical reactors and bioassay systems. By culturing vascular endothelial cells (ECs) in integrated chemical devices, vascular models have also been fabricated. Here, we utilized a thermally fused-glass microchip which is chemically and physically stable and favorable for optical detections, and cultured human arterial ECs (HAECs) in it. HAECs reached confluence within 4 days. Survival and tolerance for high shear stress $\left(25 \mathrm{dyn} / \mathrm{cm}^{2}\right)$ of the HAECs were confirmed. Furthermore, HAECs responded to inflammatory cytokine, tumor necrosis facor- $\alpha$ (TNF- $\alpha)$ and attached to more leukocyte cell line, HL-60 cells than unstimulated HAECs. Our developed device can be applied as a human arterial model, and we propose it as a new method for vascular studies.
\end{abstract}

(Received November 20, 2006; Accepted January 16, 2007; Published March 10, 2007)

\section{Introduction}

The integration of various chemical devices and complex operations onto a microchip, also known as micro total analysis systems ( $\mu$-TAS) or labs-on-a-chip, is currently a major interest for researchers due to their desirable characteristics, including reductions in reagent consumption, space requirements and analysis times. ${ }^{1,2}$ Micro-chemical devices exhibit several distinct performance advantages, including short diffusion distances, low Reynolds numbers, high interface-to-volume ratios (specific interface areas; solid/liquid or liquid/liquid) and small heat capacities. These kinds of advantages have been exploited in integrated miniaturized chemistry to produce various new, exceptionally efficient, devices that cannot be realized on a normal scale. ${ }^{3-5}$ Furthermore, as the scale of the fluidic microvolume is roughly proportional to living cell sizes and processing capabilities, combinations of cells and microchemical systems are useful to achieve practical prototypical micro-devices $^{6,7}$ such as tools for investigating cellular functions, ${ }^{8-10}$ biochemical reactors, ${ }^{11-13}$ and bio-microactuators. ${ }^{14-17}$ A microvascular model in vitro can be fabricated by applying these cell culture techniques in a microchip for vascular endothelial cells (ECs). ECs lining all blood vessels in the body have various functions to contribute to the homeostasis of the circulatory system, and they are related to a number of vascular

$\doteqdot$ To whom correspondence should be addressed.

E-mail: kitamori@icl.t.u-tokyo.ac.jp diseases, such as atherosclerosis and thrombosis. Many studies have been performed to reproduce the response of ECs under a variety of experimental conditions in vitro using various flowexposing apparatuses such as rotation disks, ${ }^{18}$ flat plates, ${ }^{19}$ and tubes. $^{20}$ Although these apparatuses can apply shear stress on ECs, micro-scale vascular structures have been difficult to reproduce. Micro-scale models of vascular systems are required because vascular diseases often occur in arterioles or capillaries. For example, diabetes induces the nonperfusion of eye capillaries, which causes blindness. To reproduce the structure and flow of such microvascular systems, a microchip is extremely useful. By exploiting a micro-fabrication technique, it is possible to fabricate a model of vascular systems from capillaries (about $5 \mu \mathrm{m}$ in diameter) to arterioles (about $200 \mu \mathrm{m}$ in diameter) on a microchip.

Generally, a microchip is made of various materials, such as glass, silicon, poly(dimethylsiloxane) (PDMS), poly(methylmethacrylate) (PMMA), other polymers, or their combination. Among these microchips, our group has mainly used a thermally fused-glass microchip because of its chemical and physical stability. ${ }^{21,22}$ Moreover, a glass microchip is favorable for optical detection under a microscope, especially under a thermal lens microscope (TLM), which is our original sensitive detector for non-fluorescent molecules in a microspace based on absorption of visible or UV light followed by photothermal process. ${ }^{21,23}$ This is because the chip can be fabricated transparency with flat surfaces, and transmit visible and UV light. Thus, various operations and detections for cell analysis can be realized on one microchip. In fact, we have so 
far developed a couple of glass microchip-based cell analysis systems in which all procedures for cell analysis, i.e., cell culture, chemical or physical stimulation and measurement including TLM detection can be performed. ${ }^{24,25}$

While there have been several reports on EC culture in microfabricated devices, most of them are made of PDMS, ${ }^{26-29}$ because they are fabricated easily and not expensive. Here, we construct a thermally fused glass microchip-based EC culture system that could be combined with various analytical operations on one microchip. To fabricate a model of vascular systems, ECs must be confluent and remain attached even when they were exposed to high shear stress, like that found in a real blood vessel. To fulfill these requirements, a glass microchannel coating method for EC culture must be optimized.

The objective of this study was to culture human arterial ECs (HAECs) in a glass microchip, and to apply them for an assay. Firstly, a condition to provide a surface coating on a glass microchannel that is sufficiently durable towards high shear stress was optimized. The coating was able to immobilize the HAECs. Secondly, HAECs were cultured in a glass microchip, and the tolerance for arterial shear stress of the HAECs was confirmed. Finally, to demonstrate that the HAEC cultured microchip is useful for an assay, a leukocyte adhesion test using inflammatory cytokine was carried out.

\section{Experimental}

\section{Preparation of HAECs}

HAECs (Kurabo) were cultured in $60 \mathrm{~mm}$ cell culture dishes at $37^{\circ} \mathrm{C}$ in $5 \% \quad \mathrm{CO}_{2}$ in an $\mathrm{EC}$ culture medium (EBM-2 supplemented with EGM-2 SingleQuots, Cambrex).

After HAECs became confluent, they were washed with $2 \mathrm{~mL}$ of buffer (HEPES, Kurabo) and detached from the cell culture dish by immersing $1 \mathrm{~mL}$ of trypsin (Kurabo) for $3 \mathrm{~min}$. Detached HAECs were added to $5 \mathrm{~mL}$ of the trypsin inhibitor (Kurabo) and the cell suspension was centrifuged at $1100 \mathrm{rpm}$ for $5 \mathrm{~min}$. Finally, the supernatant was aspirated and HAECs were re-suspended in the medium at the required concentration.

\section{Determination of the coating method}

To determine which cell adhesion protein is the best for attaching HAECs to a glass surface fast, the following slide glasses were prepared: (1) a slide glass coated with matrigel (Becton-Dickinson) after first coating with poly-L-lysine (Sigma); (2) one coated only with matrigel; (3) one coated with laminin (Sigma); (4) one coated with collagen type I (Sigma); (5) one coated with fibronectin (Sigma); and (6) one non-coated (control one). Poly-L-lysine $(0.1 \mathrm{mg} / \mathrm{mL})$ was coated at $20^{\circ} \mathrm{C}$ for $1 \mathrm{~h}$. Other proteins $(0.1 \mathrm{mg} / \mathrm{mL})$ were coated at $37^{\circ} \mathrm{C}$ in $5 \%$ $\mathrm{CO}_{2}$ for $2 \mathrm{~h}$. HAECs were prepared at a cell concentration of 1 $\times 10^{5}$ cells $/ \mathrm{mL}$ and seeded on each slide glass. Then, each glass was incubated at $37^{\circ} \mathrm{C}$ in $5 \% \mathrm{CO}_{2}$ for $2 \mathrm{~h}$. After that, each glass was washed with the medium. Each cell density was counted before and after washing. The cell attachment rates were estimated by dividing the cell density after washing by the density before washing.

\section{Fabrication of a glass microchip}

The design of a microchannel was linear. The cross section of the microchannel was semicircular with a depth of $100 \mu \mathrm{m}$ and a width of $200 \mu \mathrm{m}$. The microchannel was about $6 \mathrm{~cm}$ long.

A glass microchip was fabricated by using a photolithographic wet etching method. ${ }^{30}$ Briefly, a mechanically polished Pyrex glass substrate (bottom plate) was prepared and annealed before

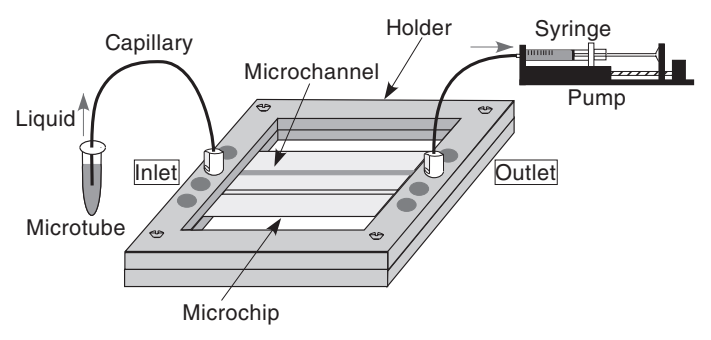

Fig. 1 Liquid introduction method into a microchannel. This system consists of a microchip, an aluminum microchip holder, a Teflon ${ }^{\mathrm{TM}}$ capillary, a microtube and a syringe equipped with a micro syringe pump. A syringe needle is connected to a capillary and a Teflon ${ }^{\mathrm{TM}}$ screw with an o-ring using epoxy glue. A Teflon ${ }^{\mathrm{TM}}$ screw is set on an outlet of the microchip which is sandwiched by a holder. Another Teflon $^{\mathrm{TM}}$ screw with a capillary is set on an inlet of the microchip and the capillary is immersed in liquid contained in the microtube. Liquid in the microtube is introduced into the microchannel by using the micro syringe pump with the withdraw mode.

use. Next, $\mathrm{Cr}$ and Au layers were evaporatively deposited on the substrate under a vacuum, and a positive photoresist was spin-coated on the metal, and UV light was exposed through a photomask. After that, the photoresist was developed, and the metal layers were etched. Then, the bare glass surface with the microchannel pattern was etched with HF solution. After glassetching, the remaining photoresist was removed. Inlet and outlet holes were drilled on another Pyrex glass substrate (cover plate). Finally, the plates were thermally bonded in a furnace.

\section{Liquid introduction method into a microchannel}

The liquid introduction method is described in Fig. 1. By using a microfluidic technique, a continuous flow system was designed and constructed. The flow rates of the liquids were controlled by syringes (Terumo) and micro syringe pumps (KD Scientific) with the withdraw mode, because air bubbles that cause serious damage to the cells were often found in the microchannel with the injection mode. All liquids (cell adhesion protein solution, cell suspension, reagents, and medium) were introduced into the microchannel by this method. The most important advantage of this method is that the contamination rate is lower than that of our conventional method (immersing a microchip with a holder in the medium). ${ }^{31}$ This is because almost all of the liquids are confined in the system.

\section{Cell culture in a microchip}

Before cell introduction, a microchannel surface was modified by cell adhesion proteins (described later). After a modification, the microchannel and the capillaries were filled with the medium.

A HAEC suspension was prepared at a cell concentration of $1 \times 10^{7}$ cells $/ \mathrm{mL}(30 \mu \mathrm{L})$, and it was introduced into the microchannel by drawing a syringe manually. After HAECs were introduced, a capillary was pinched by a clip to stop the flow in the microchannel. Then, the system was incubated at $37^{\circ} \mathrm{C}$ in $5 \% \mathrm{CO}_{2}$ for $2 \mathrm{~h}$ to allow HAECs to attach to the microchannel wall. Finally, the medium was supplied in the microchannel using a micro syringe pump at the flow rate of $0.2 \mu \mathrm{L} / \mathrm{min}$.

HAECs cultured in a microchannel were observed using a phase contrast microscope (ELIPSE TS100, Nikon) with an objective lens $(10 \times, 0.25$-NA), and the images were recorded by a digital camera (COOLPIX 4500, Nikon). Pictures of the microchannel were taken at $0 \mathrm{~h}$ (just after introducing cells), $2 \mathrm{~h}$ (just before pumping in medium), 48 and $96 \mathrm{~h}$ after introducing cells. 


\section{Nuclei and mitochondria staining}

Nuclei and mitochondria in HAECs were stained and fluorescently observed by the following procedures.

Nuclei of HAECs were stained by Hoechst 33342 (Molecular Probes). A Hoechst solution $(100 \mathrm{mg} / \mathrm{mL})$ was introduced in the microchannel at a flow rate of $0.6 \mu \mathrm{L} / \mathrm{min}$ at $37^{\circ} \mathrm{C}$ in $5 \%$ $\mathrm{CO}_{2}$ for $30 \mathrm{~min}$. Then, the microchannel was washed with a fresh medium at a flow rate of $0.6 \mu \mathrm{L} / \mathrm{min}$ for $30 \mathrm{~min}$ to wash out the Hoechst solution.

Mitochondria of HAECs were stained using a $10 \mu \mathrm{M}$ solution of rhodamine 1,2,3 (Sigma). Methods for staining were the same as those for nuclei staining.

Microchannels were observed in situ using a fluorescent microscope (IX 70, Olympus) with an objective lens (10×, 0.25NA). For nuclei observations, the excitation and emission wavelengths were 350 and $465 \mathrm{~nm}$, respectively (using a DAPI filter set). For mitochondria observations, the excitation and emission wavelengths were 492 and $535 \mathrm{~nm}$, respectively (using a FITC filter set). The images were recorded through a CCD camera (CoolSnap Photometrics, Olympus).

\section{Leukocyte adhesion assay}

To activate HAECs cultured confluently in a microchannel, $100 \mathrm{ng} / \mathrm{mL}$ of recombinant human tumor necrosis factor- $\alpha$ (TNF- $\alpha$, Sigma) in the medium without any growth factor (only EBM-2, Cambrex) were introduced at a flow rate of $0.6 \mu \mathrm{L} / \mathrm{min}$ at $37^{\circ} \mathrm{C}$ in $5 \% \mathrm{CO}_{2}$ for $4 \mathrm{~h}$. After that, the microchannel was washed with this medium under the same conditions for $30 \mathrm{~min}$ before adding a leukocyte cell line, HL-60 cells. For control experiments, two other types of microchannels were also prepared. One was a microchannel in which only HAECs were cultured (unstimulated by TNF- $\alpha$ ). Another was a blank microchannel (only coated with cell adhesion protein).

A HL-60 cells (lymphoblast-like, provided by Cell Resource Center for Biomedical Research Institute of Development, Aging and Cancer, Tohoku University, Japan) were cultured in RPMI 1640 (Sigma) supplemented with 10\% fetal bovine serum (FBS). Before use, HL-60 cells were fluorescently labeled in RPMI 1640 with $10 \mu \mathrm{M}$ CellTracker Green CMFDA (Molecular Probes) for $30 \mathrm{~min}$ at $37^{\circ} \mathrm{C}$, washed, and resuspended in EBM-2 supplemented with EGM-2 SingleQuots. The HL-60 cell suspension was controlled at the same concentration as found in human blood $\left(2 \times 10^{6}\right.$ cells $\left./ \mathrm{mL}\right){ }^{32}$

The HL-60 cell suspension was then introduced manually into the three types of microchannels and incubated at $37^{\circ} \mathrm{C}$ for 10 min to allow for attachment. Following exposure to HL-60 cells, microchannels were washed with EBM-2 supplemented with EGM-2 SingleQuots at a flow rate of $0.6 \mu \mathrm{L} / \mathrm{min}$ at $37^{\circ} \mathrm{C}$ in $5 \% \mathrm{CO}_{2}$ for $30 \mathrm{~min}$ to remove any HL-60 cells that did not attach firmly to the HAECs. The number of adherent HL-60 cells in the microchannels was determined from fluorescent images of the microchannels. Microchannels were observed in situ by using the same fluorescent microscope and objective lens described in the previous section. A FITC filter set was used here.

\section{Results and Discussion}

\section{Results of the glass coating experiment}

Generally, when cells are cultured in a microchannel, medium must flow in it for life support after cell attachment. In our previous work culturing hepatocytes in a microchannel, the medium must start to flow within $2 \mathrm{~h}$ after introducing cells. ${ }^{31}$ Therefore, it is important to attach cells to the glass surface

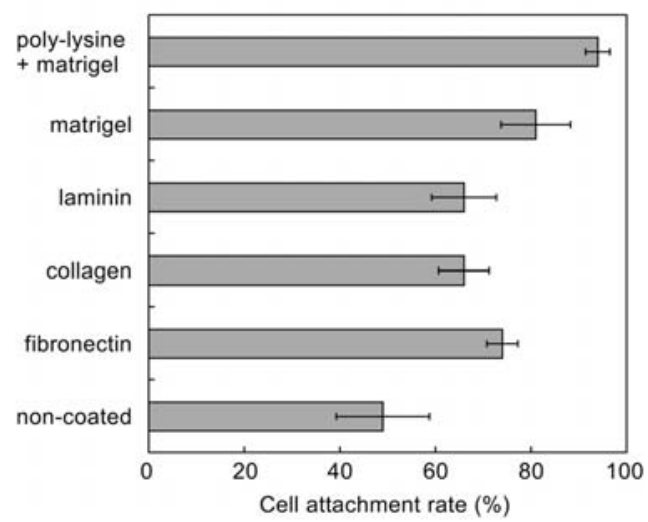

Fig. 2 Cell attachment rates $2 \mathrm{~h}$ after seeding for 6 coating conditions. Cell attachment rates were estimated by dividing the cell density after washing by the density before washing $2 \mathrm{~h}$ after seeding. Values are means \pm S.D.; $n=3$.

quickly. To determine which cell adhesion protein is useful for attaching HAECs to the glass surface quickly and is sufficiently durable towards high shear stress, we measured the cell attachment rates to slide glasses coated with a number of cell adhesion proteins $2 \mathrm{~h}$ after cell seeding.

The cell attachment rate for each cell adhesive protein is plotted in Fig. 2. Two hours after seeding, the medium flushed unattached HAECs. From the result, "poly-L-lysine + matrigel (cell attachment rate of a slide glass coated with matrigel after coating with poly-L-lysine)" was found to have the largest cell attachment rate (94\%) among the 6 types of coating conditions. Therefore, we chose "poly-L-lysine + matrigel" for adhesion protein coating of the microchannel wall.

Microchannels were coated by a similar method as that for the slide glass. Poly-L-lysine was introduced into a microchannel. After waiting for $1 \mathrm{~h}$ at $20^{\circ} \mathrm{C}$, we introduced the matrigel and incubated the microchip at $37^{\circ} \mathrm{C}$ in $5 \% \mathrm{CO}_{2}$ for $2 \mathrm{~h}$. Subsequent procedures for cell introduction and culturing are described in the Cell culture in a microchip section.

\section{Observation in a microchannel by phase contrast microscopy}

After introducing HAECs in a microchannel, we observed them periodically to confirm whether they attached to the surface and became confluent. Actual pictures of HAECs in the microchannel are shown in Fig. 3a. At 0 h, HAECs were roundshaped and were not attached to the microchannel wall. After 2 $\mathrm{h}$, HAECs attached to the microchannel wall and began to elongate. After $48 \mathrm{~h}$, HAECs spread all over the microchannel. The number of HAECs increased continuously and became confluent within $96 \mathrm{~h}$. HAECs survived in this state for about 3 weeks. From these results, we decided to use HAECs 4 days after introducing them into a microchannel for further experiments.

This is the first demonstration of a confluent HAEC culture in a thermally fused-glass microchannel. While some similar studies, such as the culture of human umbilical vein ECs (HUVECs) in a glass microchannel ${ }^{33}$ and the culture of bovine aortic ECs (BAECs) in a silicon microchannel ${ }^{34}$ were reported previously, these microchannels were formed by sealing the microfabricated devices using lids after cell culture. This method may cause cell damage when lids are attached to the devices. This problem does not occur in our method. 
(a)
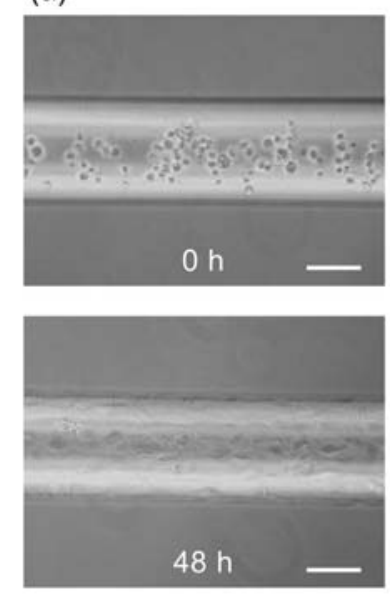

(b)

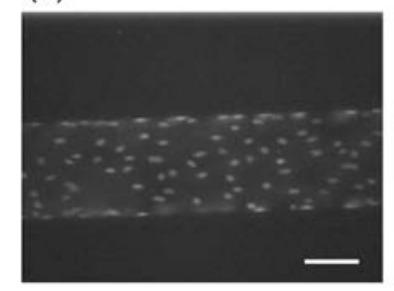

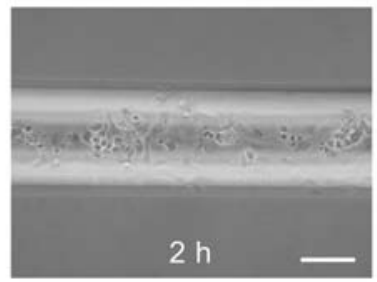

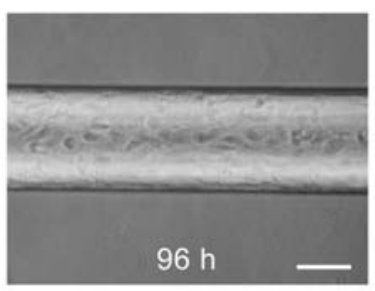

(c)

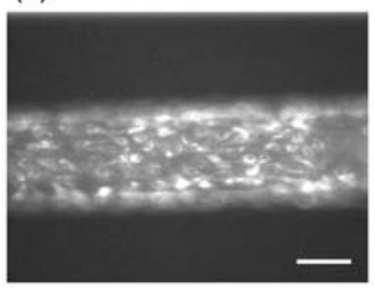

Fig. 3 Actual photos of HAECs cultured in a glass microchannel. (a) HAECs observed using a phase contrast microscope at $0 \mathrm{~h}$ (just after introducing cells), $2 \mathrm{~h}$ (just before pumping medium), 48 and 96 $\mathrm{h}$ after introducing cells. (b) A fluorescent image of nuclei stained HAECs after culturing them for 4 days in a microchannel. (c) A fluorescent image of mitochondria stained HAECs after culturing them for 4 days in a microchannel. All scale bars; $100 \mu \mathrm{m}$.

Fluorescent observation of nuclei and mitochondria stained cells After HAECs in a microchannel became confluent (4 days after introduction), we stained the nuclei and mitochondria in the HAECs to observe the cell distribution and to confirm the cell survival in the microchannel.

A photograph of nuclei stained HAECs after being cultured for 4 days in a microchannel is shown in Fig. 3b. It is readily apparent from the photo that the HAECs attached to the microchannel walls confluently and uniformly including on the microchannel edges.

Figure $3 \mathrm{c}$ shows a photograph of mitochondria stained HAECs after being cultured for 4 days in a microchannel. Since only living cells emit fluorescence, HAECs in the microchannel were surviving.

Furthermore, to confirm that HAECs remain attached to the microchannel wall and survive even when they are exposed to high shear stress like that found in an artery, we fluorescently observed mitochondria stained HAECs while shear stress was gradually increased. The shear stress against flow rate in a semicircular shaped microchannel was calculated from:

$$
\tau=\frac{6 \mu Q}{a^{2} b}
$$

where $\tau$ is the shear stress, $\mu$ is the viscosity of the medium, $Q$ is the volume flow rate, $a$ is the depth of the microchannel, and $b$ is the width of the microchannel. Here, $\mu$ was approximated as the viscosity of water at $37^{\circ} \mathrm{C}\left(0.007 \mathrm{dyn} \cdot \mathrm{s} / \mathrm{cm}^{2}\right)$. From the microchannel dimensions, $a$ was $100 \mu \mathrm{m}$ and $b$ was $200 \mu \mathrm{m}$.

When HAECs were cultured, $Q$ was kept at $0.2 \mu \mathrm{L} / \mathrm{min}$, and $\tau$ was calculated as $0.07 \mathrm{dyn} / \mathrm{cm}^{2}$ from Eq. (1). After mitochondria in HAECs were stained, $Q$ was gradually increased to $70 \mu \mathrm{L} / \mathrm{min}\left(\tau=25 \mathrm{dyn} / \mathrm{cm}^{2}\right)$ during $15 \mathrm{~min}$ and kept at this volume flow rate for $10 \mathrm{~min}$.

As a result, the fluorescence intensity did not change during the observation. This meant that HAECs remained attached to the microchannel wall and survived even when they were exposed to higher shear stress $\left(25 \mathrm{dyn} / \mathrm{cm}^{2}\right)$ than that found in an artery $\left(10-23 \mathrm{dyn} / \mathrm{cm}^{2}\right),{ }^{35}$ or like that used in most of the previously reported EC cultured microchannels (less than about $\left.10 \mathrm{dyn} / \mathrm{cm}^{2}\right){ }^{26}$ These facts support that we succeeded in constructing a HAEC culture system in a thermally fused-glass microchip.

\section{Adhesion of leukocytes to HAECs}

To demonstrate that HAECs cultured in a microchannel are useful for an assay, we measured the adhesion number of HL-60 cells to HAECs with and without recombinant TNF- $\alpha$. TNF- $\alpha$ is a pleiotropic cytokine produced by a variety of cell types, including macrophages, neutrophils, lymphocytes, and ECs. It promotes leukocyte adhesion and migration at inflammatory sites. ${ }^{36}$

The fluorescent images of the three types of microchannels (HAEC cultured and TNF- $\alpha$ stimulated, HAEC cultured, blank) after introduction and washing of HL-60 cells are shown in Fig. 4a. The number of adherent HL-60 cells per $1 \mathrm{~mm}^{2}$ in microchannels was counted from these images. Each cell density is plotted in Fig. $4 \mathrm{~b}$.

These data indicated that HL-60 cells attached to a HAEC cultured microchannel stimulated by TNF- $\alpha$ numbered more than the cells attached to only HAEC cultured or blank microchannels. There were no distinct differences between only HAEC cultured or blank microchannels. From these results, we found that HAECs in microchannels were in a quiescent state and responded to cytokines.

Similar results were previously obtained using HUVECs cultured in a cylindrical, linear microtube made of collagen. ${ }^{37}$ However, that is difficult to apply for more complicated structures such as curves and branches like in actual vascular systems because of its fabrication process (drawing a needle after casting gels around the needle). By contrast, our developed glass microchannels can be fabricated for various designs.

\section{Conclusions}

In this report, we explain how we successfully cultured HAECs in a glass microchip and applied them for an assay. Firstly, we optimized a condition to provide a surface coating on a glass microchannel that is sufficiently durable towards high shear stress. Cell attachment rates $2 \mathrm{~h}$ after seeding for various adhesive proteins were compared, and the "poly-L-lysine + matrigel" coating was chosen for an adhesion protein coating on the microchannel wall. Secondly, we cultured HAECs in a glass microchip confluently and confirmed the tolerance of the HAECs towards high shear stress like that found in an artery $\left(10-23 \mathrm{dyn} / \mathrm{cm}^{2}\right) .^{35}$ HAECs remained attached to the microchannel wall and survived even when exposed to higher shear stress $\left(25 \mathrm{dyn} / \mathrm{cm}^{2}\right)$ than that. Finally, to demonstrate that the HAEC cultured microchip is useful for an assay, a leukocyte adhesion test using inflammatory cytokine was carried out. HL60 cells attached to a HAEC cultured microchannel stimulated by TNF- $\alpha$ numbered more than the cells attached to only HAEC 
(a)
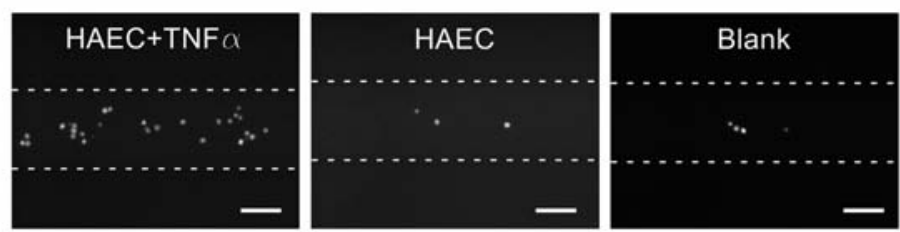

(b)

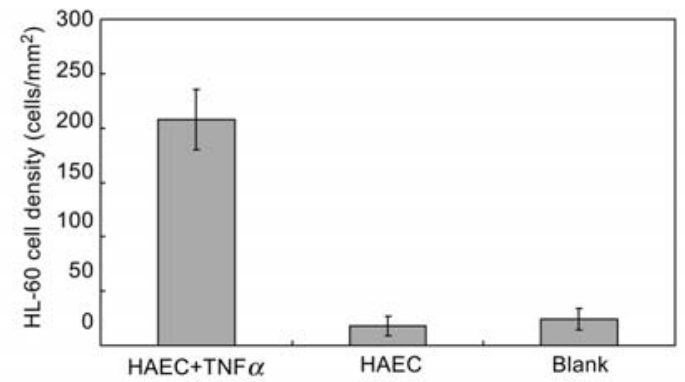

Fig. 4 Results of leukocyte adhesion assay. (a) Fluorescent images of the 3 types of microchannels (HAEC cultured and TNF- $\alpha$ stimulated, HAEC cultured, blank) after introduction and washing of HL-60 cells. All scale bars; $100 \mu \mathrm{m}$. (b) The density of attached HL-60 cells to each microchannel. Values are means \pm S.D.; $n=5$.

cultured or blank microchannels. From these results, we found that HAECs in microchannels were in a quiescent state and responded to cytokines.

This device can be incorporated into glass microchip-based total chemical analysis systems. As an example of future applications, monitoring of the time course of EC-derived nitric oxide (NO) release proportional to shear stress could be realized by combining this system with our previously developed ultrasensitive cell-derived NO detection system using TLM. ${ }^{25}$

In the further future, it could be applied to fabricate a component of a cardiovascular circulatory system micro-model to study mechanisms of circulatory physiology, pathology, and developmental biology, or for medical diagnoses of patients using cultured autologous cells.

\section{References}

1. G. M. Whitesides, Nature, 2006, 442, 368.

2. P. S. Dittrich, K. Tachikawa, and A. Manz, Anal. Chem., 2006, $78,3887$.

3. D. J. Beebe, J. S. Moore, J. M. Bauer, Q. Yu, R. H. Liu, C. Devadoss, and B.-H. Jo, Nature, 2000, 404, 588.

4. T. Thorsen, S. J. Maerkl, and S. R. Quake, Science, 2002, 298, 580 .

5. A. Groisman, M. Enzelberger, and S. R. Quake, Science, 2003, 300, 955.

6. J. El-Ali, P. K. Sorger, and K. F. Jensen, Nature, 2006, 442, 403.

7. D. N. Breslauer, P. J. Lee, and L. P. Lee, Mol. Biosyst., 2006, 2, 97.

8. S. Takayama, E. Ostuni, P. LeDuc, K. Naruse, D. E. Ingber, and G. M. Whitesides, Nature, 2001, 411, 1016.

9. E. M. Lucchetta, J. H. Lee, L. A. Fu, N. H. Patel, and R. F. Ismagilov, Nature, 2005, 434, 1134.

10. H. Lu, M. A. Schmidt, and K. F. Jensen, Lab Chip, 2005, 5, 23.

11. W. Gu, X. Zhu, N. Futai, B. S. Cho, and S. Takayama, Proc. Natl. Acad. Sci. U. S. A, 2004, 101, 15861.

12. F. K. Balagaddé, L. You, C. L. Hansen, F. H. Arnold, and S. R. Quake, Science, 2005, 309, 137.
13. A. Groisman, C. Lobo, H. Cho, J. K. Campbell, Y. S. Dufour, A. M. Stevens, and A. Levchenko, Nat. Methods, 2005, 2, 685 .

14. K. Morishima, Y. Tanaka, M. Ebara, T. Shimizu, M. Yamato, A. Kikuchi, T. Okano, and T. Kitamori, Sens. Actuators, B, 2006, 119, 345.

15. Y. Tanaka, K. Morishima, T. Shimizu, A. Kikuchi, M. Yamato, T. Okano, and T. Kitamori, Lab Chip, 2006, 6, 230.

16. Y. Tanaka, K. Morishima, T. Shimizu, A. Kikuchi, M. Yamato, T. Okano, and T. Kitamori, Lab Chip, 2006, 6, 362.

17. Y. Tanaka, K. Sato, T. Shimizu, M. Yamato, T. Okano, and T. Kitamori, Lab Chip, 2007, 7, 207.

18. T. Nozawa, C. P. Cheng, T. Noda, and W. C. Little, Circulation, 1994, 90, 3047.

19. H. J. Berger, S. K. Prasad, A. J. Davidoff, D. Pimental, O. Ellingsen, J. D. Marsh, T. W. Smith, and R. A. Kelly. Am. J. Physiol.-Heart Circul. Physiol., 1994, 266, H341.

20. Y. Xia, L. M. Buja, R. C. Scarpulla, and J. B. McMillin. Proc. Natl. Acad. Sci. U. S. A., 1997, 94, 11399.

21. T. Kitamori, M. Tokeshi, A. Hibara, and K. Sato, Anal. Chem., 2004, 76, 52A.

22. K. Sato, A. Hibara, M. Tokeshi, H. Hisamoto, and T. Kitamori, Anal. Sci., 2003, 19, 15.

23. S. Hiki, K. Mawatari, A. Hibara, M. Tokeshi, and T. Kitamori, Anal. Chem., 2006, 78, 2859.

24. E. Tamaki, K. Sato, M. Tokeshi, K. Sato, M. Aihara, and T. Kitamori, Anal. Chem., 2002, 74, 1580.

25. M. Goto, K. Sato, A. Murakami, M. Tokeshi, and T. Kitamori, Anal. Chem., 2005, 77, 2125.

26. J. W. Song, W. Gu, N. Futai, K. A. Warner, J. E. Nor, and S. Takayama, Anal. Chem., 2005, 77, 3993.

27. T. D. Oblak, P. Root, and D. M. Spence, Anal. Chem., 2006, 78, 3193.

28. P. Mulder, S. Koster, H. J. van der Linden, G. Molema, and E. Verpoorte, in Proceedings of the Micro Total Analysis Systems 2005, ed. K. F. Jensen, J. Han, D. J. Harrison, and J. Voldman, 2005, Vol. 2, Transducers Research Foundation, San Diego, 1383.

29. M. Shin, K. Matsuda, O. Ishii, H. Terai, M. Kaazempur- 
Mofrad, J. Borenstein, M. Detmar, and J. P. Vacanti, Biomed. Microdevices, 2004, 6, 269.

30. A. Hibara, M. Tokeshi, K. Uchiyama, H. Hisamoto, and T. Kitamori, Anal. Sci., 2001, 17, 89.

31. Y. Tanaka, K. Sato, M. Yamato, T. Okano, and T. Kitamori, J. Chromatogr., A, 2006, 1111, 233.

32. M. P. Bevilacqua, J. S. Pober, M. E. Wheeler, R. S. Cotran, and A. Gimbrone Jr., J. Clin. Invest., 1985, 76, 2003.

33. M. D. Frame and I. H. Sarelius, Microcirculation, 2000, 7,
419.

34. B. L. Gray, D. K. Lieu, S. D. Collins, R. L. Smith, and A. I. Barakat, Biomed. Microdevices, 2002, 4, 9.

35. A. Kamiya, R. Bukhari, and T. Togawa, Bull. Math. Biol., 1984, 46, 127.

36. C. F. Bennett, T. P. Condon, S. Grimm, H. Chan, and M.Y. Chiang, J. Immunol., 1994, 152, 3530.

37. K. M. Chrobak, D. R. Potter, and J. Tien, Microvasc. Res., 2006, $71,185$. 